\title{
Electroconvulsive Therapy as an Effective Alternative in Parkinson's Disease Associated Psychosis: A Case Report and Brief Review of the Literature
}

\author{
Sevda Sarikaya ${ }^{\mathrm{a}}$, Gokben Hizli Sayar ${ }^{\mathrm{b}, \mathrm{c}}$, Eylem Ozten $^{\mathrm{b}}$, Gul Eryilmaz
}

\begin{abstract}
The psychotic symptoms related to Parkinson's Disease (PD) are often resistant to antipsychotics, and management of psychotic features is one of the most critical issues in the treatment of PD. We reported a 42-year-old man with PD and medically intractable PD associated psychosis who was significantly benefitted from electroconvulsive therapy.
\end{abstract}

Keywords: Electroconvulsive therapy; Parkinson's Disease; Psychosis

\section{Introduction}

Psychotic symptoms (hallucinations and delusions) are among the most disabling long-term complications of Parkinson's Disease (PD) and cause significant distress for both caregiver and patient [1]. Presence of psychotic symptoms is also a risk factor for the development of PD dementia and mortality [2, 3]. Recently, new criteria for PD associated psychosis were proposed by an NIH-sponsored workshop. According to these criteria, the diagnosis of PDAP requires at least one of the following features: hallucinations, delusions, sense of presence and visual illusions, occurring with a clear sensorium and a chronic course [4]. In a study patients $43 \%$ of $116 \mathrm{PD}$ patients had either hallucinations or delusions, while $60 \%$ fulfilled the NINDS-NIMH criteria for PD associated psychosis [5].

These psychotic symptoms are likely to be induced by high doses of antiparkinsonian drugs, but they also can oc-

\footnotetext{
Manuscript accepted for publication January 14, 2014

${ }^{a}$ Beykoz Kavaclk Medistate Hospital, Istanbul, Turkey

${ }^{b}$ Uskudar University, Istanbul, Turkey

${ }^{\mathrm{c}}$ Corresponding author: Gokben Hizli Sayar, Uskudar University,

Istanbul, Turkey. Email: gokben.hizlisayar@uskudar.edu.tr
}

doi: http://dx.doi.org/10.14740/jmc1646w cur intrinsically [6]. Presence of psychotic symptoms significantly alters prognosis [7].

The psychotic symptoms are often resistant to antipsychotics, and PD patients easily become intolerant to them [8]. Thus, psychosis is an essential determinant of PD prognosis, and its management is one of the most critical issues in the management of PD. There are a few studies about electroconvulsive therapy (ECT) use in PD psychosis and some other non-motor symptoms. Herein, we present a case of PD who had benefitted from ECT against psychotic and motor symptoms.

\section{Case Report}

A 42-year-old male patient, who was diagnosed as PD according to United Kingdom Parkinson's Disease Society Brain Bank clinical diagnostic criteria [9] since 2008, attended to our clinic with a history of agitation, paranoid delusions and severe insomnia. He was on a treatment of Ropinirole $12 \mathrm{mg}$ /day. Neuropsychological evaluation revealed no cognitive impairment, but just mild memory impairment comprising of secondary attention deficits without primary memory deficits. He had persecutive and paranoid delusions with no insight, and had depressive periods which were not long enough to be diagnosed as depression. His delusions were jealous type, believing that his spouse is cheating in the absence of evidence to support his belief. He also had visual hallucinations as vivid sensation of the presence of a man somewhere in the house. He also reported that he heard his wife had sexual intercourse with that man while the patient was sleeping beside them. Neurological examination did not demonstrate any resting tremor but moderate rigidity of both upper extremities. His axial rigidity was prominent. He also had bradykinesia and micrographia.

After the clinical evaluation, he was diagnosed as PD associated psychosis he was started on quetiapine $25 \mathrm{mg}$ /day with a plan to increase the dose gradually up to $100 \mathrm{mg} /$ day. For the control of mild depressive symptoms, escitalopram $10 \mathrm{mg} /$ day was also added to his treatment. He was scored 4 on the Hoehn and Yahr Scale (HYS), 82 on Positive and Negative Syndrome Scale for Schizophrenia (PANSS) and 
47 on Brief Psychiatric Rating Scale (BPRS).

Ten days later, his psychotic symptoms worsened and eventually lead to hospital admission. His aggression got worsened and his homicide thoughts became more evident. He got a gun for punishing his wife. Upon his admission to the inpatient unit, his medication was gradually switched to clozapine $100 \mathrm{mg} /$ day as the quetiapine dose tapered off. Escitalopram $10 \mathrm{mg}$ /day and ropinirole $12 \mathrm{mg} /$ day were continued and ECT planned. He received a total of five sessions of ECT, performed on an every other day basis with Thymartron System IV (Somatics, IL, USA). Bitemporal bilateral electrode placement was selected. Anesthesia induction was attained with methohexital 100 - $150 \mathrm{mg}$ IV and succinylcholine 100 - $120 \mathrm{mg}$ IV. Seizure threshold was determined using a dose titration schedule. His motor and psychotic signs showed significant improvement two weeks after. His escitalopram plasma level was found to be $38 \mathrm{ng} / \mathrm{mL}$ $(15-80 \mathrm{ng} / \mathrm{mL})$, clozapine and norclozapine plasma levels were $124.9 / 144.0 \mathrm{ng} / \mathrm{mL}(350-600 \mathrm{ng} / \mathrm{mL})$. Even though the plasma levels of clozapine were below the therapeutic levels, it was not needed to change the dosage due to his near perfect clinical improvement. His scores on HYS, PANSS and BPRS were 2, 38 and 18 respectively.

Last follow-up appointment with the patient was 6 months after the last ECT and, his scores almost remained the same. Patient is still in our follow-up with planned clinical visits every 4 months.

\section{Discussion}

Researchers are increasingly attending to and characterizing the non-motor symptoms of the disease such as depression, apathy, dementia, insomnia and psychosis [10]. Thus, PD is no longer conceptualized as a sole motor disorder.

Previous studies have repeatedly shown that several clinical factors were associated with visual hallucinations and PD associated psychosis, most consistently found being dementia, a longer duration of PD, and/or an older age [11]. In our case, age was extremely young, PD duration was 5 years and there was not any dementia. In this case dopaminergic treatment was suspected as the cause of the psychosis, but reduction on ropinirole dose or switching to another medication was not possible due to worsening of motor symptoms. He responded well to ECT for psychotic symptoms and motor symptoms.

Initial management of psychosis in PD requires exclusion of other organic causes of psychosis such as psychoactive substances, intoxication, medication side effects and endocrine disorders. Delirium should also be excluded if symptoms start acutely [12]. In treatment of PD associated psychosis, anticholinergic medication and dopamine agonists should be reduced. But this dose reduction may worsen the motor symptoms. Risperidone and typical antipsychotics should be avoided due to their increased propensity to cause extrapyramidal side-effects and worsening of motor function. Generally atypical antipsychotics often used initially due to their low risk of extrapyramidal side effects. Quetiapine is usually relatively well tolerated, but it did not work in our case. Olanzapine is an effective choice but it can cause more adverse effects on motor function than quetiapine [1]. Aripiprazole and ziprasidone were also suggested as effective and relatively safe treatment options in PD associated psychosis $[13,14]$. Clozapine should be used cautiously in view of the risk of agranulocytosis and there is a mandatory requirement for blood monitoring [15].

In the literature, there are some studies demonstrating the role of ECT in controlling motor symptoms of PD. The first study describing the use of ECT in the motor symptoms of PD was published in 1947, and since then, it has been suggested in some case reports of patients with PD and comorbid psychiatric illnesses treated with ECT, showing secondary improvement of the motor symptoms $[16,17]$. In a study of Usui et al, the authors mentioned about a course of ECT producing notable improvements not only in PD associated psychosis but also in the severity of PD. The findings of change in regional cerebral blood flow after electroconvulsive therapy suggested implications for dysfunction in the middle frontal region for patients with PD psychosis [18]. In another case on a patient with PD and obsessive compulsive disorder (OCD). After the ECT, his OCD symptoms and mood improved [19]. There are few studies on implementation of ECT on patients with deep brain stimulator (DBS), which offer additional evidence about the safety and efficacy of ECT in patients with DBS [20].

Although there is a case report with schizoaffective disorder and anticholinergic refractory neuroleptic-induced parkinsonism manifested a marked increase of parkinsonian symptoms and dystonia after ECT [21], the majority of the published articles suggested that ECT can be used to treat PD associated psychosis, depression, OCD and even though motor symptoms.

\section{Conclusion}

Psychosis in PD is common and associated with a range of negative outcomes. Psychosis is a risk factor for the development of PD dementia and mortality. ECT should be considered for PD patients with problems not responsive to medications. However, ECT can increase the risk of delirium, particularly in patients with cognitive impairment.

\section{Declaration}

The authors declare no competing interest. No financial support was received for this paper. Authors would like to thank Basar Sarikaya, M.D. for language editing. 


\section{References}

1. Zahodne LB, Fernandez HH. Parkinson's psychosis. Curr Treat Options Neurol. 2010;12(3):200-211.

2. Factor SA, Feustel PJ, Friedman JH, Comella CL, Goetz CG, Kurlan R, Parsa M, et al. Longitudinal outcome of Parkinson's disease patients with psychosis. Neurology. 2003;60(11):1756-1761.

3. Forsaa EB, Larsen JP, Wentzel-Larsen T, Alves G. What predicts mortality in Parkinson disease?: a prospective population-based long-term study. Neurology. 2010;75(14):1270-1276.

4. Ravina B, Marder K, Fernandez HH, Friedman JH, McDonald W, Murphy D, Aarsland D, et al. Diagnostic criteria for psychosis in Parkinson's disease: report of an NINDS, NIMH work group. Mov Disord. 2007;22(8):1061-1068.

5. Fenelon G, Soulas T, Zenasni F, Cleret de Langavant L. The changing face of Parkinson's disease-associated psychosis: a cross-sectional study based on the new NINDSNIMH criteria. Mov Disord. 2010;25(6):763-766.

6. Ueda S, Koyama K, Okubo Y. Marked improvement of psychotic symptoms after electroconvulsive therapy in Parkinson disease. J ECT. 2010;26(2):111-115.

7. Friedman JH. Parkinson disease psychosis: Update. Behav Neurol. 2013;27(4):469-477.

8. Rabey JM. Hallucinations and psychosis in Parkinson's disease. Parkinsonism Relat Disord. 2009;15(Suppl 4):S105-110.

9. Hughes AJ, Daniel SE, Kilford L, Lees AJ. Accuracy of clinical diagnosis of idiopathic Parkinson's disease: a clinico-pathological study of 100 cases. J Neurol Neurosurg Psychiatry. 1992;55(3):181-184.

10. Gregory P, Morgan K, Lynall A. Improving sleep management in people with Parkinson's. Br J Community Nurs. 2012;17(1):14-18, 20.

11. Latoo J, Mistry M, Dunne FJ. Psychosis in Parkinson's disease: diagnosis and management. Progress in Neurology and Psychiatry. 2012;5:7-10.
12. Lee AH, Weintraub D. Psychosis in Parkinson's disease without dementia: common and comorbid with other non-motor symptoms. Mov Disord. 2012;27(7):858863.

13. Lopez-Meza E, Ruiz-Chow A, Ramirez-Bermudez J. Aripiprazole in psychosis associated with Parkinson's disease. J Neuropsychiatry Clin Neurosci. 2005;17(3):421422.

14. Duran-Ferreras E, Alvarez-Lopez M, Garcia-Moreno JM, Chacon J. [Ziprasidone in Parkinsonian dopamine psychosis]. Rev Neurol. 2008;46(8):476-480.

15. Fuchs GA. Psychoses in idiopathic Parkinson's disease - risk factors in the course of disease. Psychoneuro. 2008;34:361-366.

16. Pintor LP, Valldeoriola F, Fernandez-Egea E, Sanchez R, Rami L, Tolosa E, Muniz A, et al. Use of electroconvulsive therapy in Parkinson disease with residual axial symptoms partially unresponsive to L-dopa: a pilot study. J ECT. 2012;28(2):87-91.

17. Muralidharan K, Thimmaiah R, Chakraborty V, Jain S. Bifrontal ECT for drug-induced psychosis in Parkinson's disease. Indian J Psychiatry. 2011;53(2):156-158.

18. Usui C, Hatta K, Doi N, Kubo S, Kamigaichi R, Nakanishi A, Nakamura H, et al. Improvements in both psychosis and motor signs in Parkinson's disease, and changes in regional cerebral blood flow after electroconvulsive therapy. Prog Neuropsychopharmacol Biol Psychiatry. 2011;35(7):1704-1708.

19. Bailine S, Kremen N, Kohen I, Linder H, Schwartz GJ, Mogilner AY, Pourfar M. Bitemporal electroconvulsive therapy for depression in a Parkinson disease patient with a deep-brain stimulator. J ECT. 2008;24(2):171172.

20. Gadit AM, Smigas T. Efficacy of ECT in severe obsessive-compulsive disorder with Parkinson's disease. BMJ Case Rep. 2012;2012.

21. Hanin B, Lerner Y, Srour N. An unusual effect of ECT on drug-induced parkinsonism and tardive dystonia. Convuls Ther. 1995;11(4):271-274. 